\title{
Factorization, reduction and embedding in integrable cellular automata
}

\author{
Atsuo Kuniba* Taichiro Takagi† and Akira Takenouchi*
}

\begin{abstract}
Factorized dynamics in soliton cellular automata with quantum group symmetry is identified with a motion of particles and anti-particles exhibiting pair creation and annihilation. An embedding scheme is presented showing that the $D_{n}^{(1)}$-automaton contains, as certain subsectors, the box-ball systems and all the other automata associated with the crystal bases of non-exceptional affine Lie algebras. The results extend the earlier ones to higher representations by a certain reduction and to a wider class of boundary conditions.
\end{abstract}

\section{Introduction}

In [HKT1], a class of one dimensional cellular automata with quantum group symmetry were introduced. They are associated with the crystal bases $[\mathrm{KKM}]$ of non-exceptional affine Lie algebras $A_{2 n-1}^{(2)}, A_{2 n}^{(2)}, B_{n}^{(1)}, C_{n}^{(1)}, D_{n}^{(1)}$ and $D_{n+1}^{(2)}$. Since then, (i) configurations that behave as solitons are extracted [HKT1], (ii) scattering of solitons is determined in terms of combinatorial $R$ by utilizing the quantum group symmetry [HKOTY], (iii) time evolution under a sufficiently large carrier is factorized into a product of Weyl group operators [HKT2], (iv) the resulting factorized dynamics is described by an elementary algorithm using particles and anti-particles [HKT3], (v) tropical $R$ for geometric crystals is formulated [KOTY1], which yileds the evolution equation of the automata under ultradiscretization [TTMS], (vi) the tropical $R$ is bilinearized in terms of tau functions of the DKP hierarchy [KOTY2], allocating the automata in classical integrable systems.

Through the developments these automata have now been understood at a level comparable with the well known box-ball systems [TS, T, TM] corresponding to $A_{n}^{(1)}$ [HHIKTT, FOY]. The factorization of the time evolution in this case was known as the decomposition into a finer process where one only moves the balls with a fixed color.

The states of our automata have the form (1), where $a$ specifies the boundary condition and $l=\left(l_{i}\right)_{i \in \mathbb{Z}}$ denotes the 'capacities of the aligned boxes' in the terminology of the box-ball systems and 'magnitude of local spins' in the terminology of solvable lattice models. Throughout the paper the automata with $\left(\forall l_{i}=1\right)_{i \in \mathbb{Z}}$ and general $\left(l_{i}\right)_{i \in \mathbb{Z}}$ will be called basic and inhomogeneous, respectively.

The goal of this paper is to provide the full generalization of the above result (iv) which was limited to the basic automata with the boundary condition $a=1$. Our approach involves three essential ingredients; factorization (section 2 ), reduction (section 3) and embedding (section 4). Factorization of the time evolution into the Weyl

*Institute of Physics, University of Tokyo, Komaba, Tokyo 153-8902, Japan

${ }^{\dagger}$ Department of Applied Physics, National Defense Academy, Kanagawa 239-8686, Japan 
group operators had been proved for any inhomogeneous automata associated with $A_{n}^{(1)}, A_{2 n-1}^{(2)}, A_{2 n}^{(2)}, B_{n}^{(1)}, C_{n}^{(1)}, D_{n}^{(1)}$ and $D_{n+1}^{(2)}$ in [HKT2].

In sections 2 and 3 we concentrate on the $D_{n}^{(1)}$ case and the former is devoted to the quotation of the result in [HKT2]. Section 3 contains our main Theorem 7, which claims that under any boundary condition $a$, the factorized dynamics of the inhomogeneous $D_{n}^{(1)}$-automaton admits a reduction to the basic one except inserting a certain 'reshuffling operator' $Q$. See Examples 4 and 5. It integrates the crystal basis theory on the time evolution into the simple diagrams (8), which represent a motion of particles and anti-particles that undergo pair creation and annihilation. Until this point we achieve double simplifications of the inhomogeneous $D_{n}^{(1)}$-automaton; first by the factorization with respect to 'color' of the particles and second by the reduction to the basic case up to reshuffling. They make the time evolution a handy enough calculation and suit programming due to the locality of the diagrams (8).

In section 4 we explain the embedding, by which the $D_{n}^{(1)}$-automaton can be regarded as a 'master automaton' that contains, as certain subsectors, all the other inhomogeneous automata associated with $A_{n}^{(1)}, A_{2 n-1}^{(2)}, A_{2 n}^{(2)}, B_{n}^{(1)}, C_{n}^{(1)}$ and $D_{n+1}^{(2)}$. In a sense this is the third simplification that unites the number of results obtained previously. Our embedding scheme is a synthesis of the preceeding ones in [HKOTY] and [KOTY2].

In the box-ball system associated with $A_{n-1}^{(1)}$, all the colors are equivalent under $\mathbb{Z}_{n}$ symmetry. Such a symmetry is absent in the relevant crystal graph for $D_{n}^{(1)}$ in Figure 1 in appendix A. This motivates us to study the automaton under a general boundary condition specified by any point $a$ in the crystal graph. Since time evolutions under any boundary conditions are connected by a similarity transformation (see Definition 1), qualitative features of the automata remains the same. However, solitons show up in various guises depending on $a$. Section 6 contains the list of such configurations under the general boundary condition.

We defer the technical details to section 5 and appendix B, where a proof of Theorem 7 is outlined. The key is to identify the crystal theoretic Weyl group operator $S_{i}[\mathrm{~K}]$ with the particle motion operator $K_{j}$ via a certian gauge transformation as in Proposition 11 and Lemma 18. We note that for the box-ball systems, a reduction to the basic case was also known in [HHIKTT, F].

\section{Factorization in inhomogeneous $D_{n}^{(1)}$-automaton}

For a positive integer $k$, let $B_{k}$ be the crystal for $k$-fold symmetric tensor representation of $D_{n}^{(1)}(n \geq 4)[\mathrm{KKM}]$. Each element of $B_{k}$ is written as $\vec{x}=\left(x_{1}, \ldots, x_{n}, x_{-n}, \ldots, x_{-1}\right)$, where $x_{i}$ 's are non-negative integers that add up to $k$ and satisfy the condition $x_{n} x_{-n}=0$. Let $J=\{ \pm 1, \ldots, \pm n\}$. For $a \in J$ we let $\vec{a} \in B_{k}$ denote the unique element characterized by $x_{a}=k$. (We suppress the dependence on $k$.) Let $l=\left(l_{i}\right)_{i \in \mathbb{Z}}$ be an array of positive integers. Given $a \in J$ and $l=\left(l_{i}\right)_{i \in \mathbb{Z}}$, recall the inhomogeneous $D_{n}^{(1)}$-automaton [HKT2] having the capacity $l$ and the prescribed boundary condition $a$. It is a discrete dynamical system over the set

$$
W[a]_{l}=\left\{\left(\ldots, \vec{b}_{i}, \vec{b}_{i+1}, \ldots\right) \in \cdots \times B_{l_{i}} \times B_{l_{i+1}} \times \cdots \mid \vec{b}_{i}=\vec{a} \text { for }|i| \gg 1\right\},
$$

where the product $\times$ of sets actually means the tensor product of the crystals $B_{l_{i}}$.

Let $S_{0}, \ldots, S_{n}$ be the Weyl group operators $[\mathrm{K}]$ acting on $\coprod_{a \in J} W[a]_{l}$. They satisfy the Coxeter relations, which include in particular $S_{i}^{2}=i d, S_{0} S_{1}=S_{1} S_{0}$ and 
$S_{n} S_{n-1}=S_{n-1} S_{n}$. We introduce the operator $\sigma^{\Delta}$ that acts on $\boldsymbol{b}=\left(\ldots, \vec{b}_{i}, \vec{b}_{i+1}, \ldots\right) \in$ $\coprod_{a \in J} W[a]_{l}$ as $\sigma^{\Delta}(\boldsymbol{b})=\left(\ldots, \sigma\left(\vec{b}_{i}\right), \sigma\left(\vec{b}_{i+1}\right), \ldots\right)$, where $\sigma\left(\vec{b}_{i}\right)$ is obtained from $\vec{b}_{i}=$ $\left(x_{1}, \ldots, x_{n}, x_{-n}, \ldots, x_{-1}\right) \in B_{l_{i}}$ by the simultaneous interchanges $x_{1} \leftrightarrow x_{-1}$ and $x_{n} \leftrightarrow$ $x_{-n}$. It induces the involutive automorphism $\sigma^{\Delta} S_{0} \sigma^{\Delta}=S_{1}, \sigma^{\Delta} S_{n-1} \sigma^{\Delta}=S_{n}$ and $\sigma^{\Delta} S_{i} \sigma^{\Delta}=S_{i}$ for $i \neq 0, n$.

In terms of these operators the time evolution $\mathcal{T}_{a}: W[a]_{l} \rightarrow W[a]_{l}$ of our $D_{n}^{(1)}-$ automaton is defined as follows.

\section{Definition 1.}

$$
\begin{aligned}
& \mathcal{T}_{1}=\sigma^{\Delta} S_{0} S_{2} S_{3} \cdots S_{n-2} S_{n} S_{n-1} \cdots S_{2} S_{0}, \\
& \mathcal{T}_{a}=S_{a-1}^{-1} \mathcal{T}_{a-1} S_{a-1} \quad(\text { for } \quad 2 \leq a \leq n) \\
& = \begin{cases}\sigma^{\Delta} S_{a} S_{a+1} \cdots S_{n-2} S_{n} S_{n-1} \cdots S_{2} S_{0} S_{1} \cdots S_{a-1} & 2 \leq a \leq n-1, \\
\sigma^{\Delta} S_{n-1} S_{n-2} \cdots S_{2} S_{0} S_{1} \cdots S_{n-1} & a=n,\end{cases} \\
& \mathcal{T}_{-n}=S_{n}^{-1} \mathcal{T}_{n-1} S_{n}=\sigma^{\Delta} S_{n} S_{n-2} \cdots S_{2} S_{0} S_{1} \cdots S_{n-2} S_{n}, \\
& \mathcal{T}_{-a}=S_{a}^{-1} \mathcal{T}_{-a-1} S_{a} \quad(\text { for } \quad 1 \leq a \leq n-1) \\
& = \begin{cases}\sigma^{\Delta} S_{a-1} S_{a-2} \cdots S_{2} S_{0} S_{1} \cdots S_{n-2} S_{n} S_{n-1} \cdots S_{a} & 2 \leq a \leq n-1, \\
\sigma^{\Delta} S_{1} S_{2} S_{3} \cdots S_{n-2} S_{n} S_{n-1} \cdots S_{2} S_{1} & a=1 .\end{cases}
\end{aligned}
$$

These operators can be extended to $\coprod_{a \in J} W[a]_{l}$, where $\mathcal{T}_{a}=\left(\mathcal{T}_{-a}\right)^{-1}$ is valid for any $1 \leq a \leq n$. See also (20) and (21) for a property of the Weyl group elements.

Example 2. For $n=4, \mathcal{T}_{a}$ 's read

$$
\begin{aligned}
& \mathcal{T}_{1}=\sigma^{\Delta} S_{0} S_{2} S_{4} S_{3} S_{2} S_{0}, \\
& \mathcal{T}_{2}=\sigma^{\Delta} S_{2} S_{4} S_{3} S_{2} S_{0} S_{1}, \\
& \mathcal{T}_{3}=\sigma^{\Delta} S_{4} S_{3} S_{2} S_{0} S_{1} S_{2}, \\
& \mathcal{T}_{4}=\sigma^{\Delta} S_{3} S_{2} S_{0} S_{1} S_{2} S_{3}, \\
& \mathcal{T}_{-4}=\sigma^{\Delta} S_{4} S_{2} S_{0} S_{1} S_{2} S_{4}, \\
& \mathcal{T}_{-3}=\sigma^{\Delta} S_{2} S_{0} S_{1} S_{2} S_{4} S_{3}, \\
& \mathcal{T}_{-2}=\sigma^{\Delta} S_{0} S_{1} S_{2} S_{4} S_{3} S_{2}, \\
& \mathcal{T}_{-1}=\sigma^{\Delta} S_{1} S_{2} S_{4} S_{3} S_{2} S_{1} .
\end{aligned}
$$

The automaton was originally introduced in [HKT1] for $\forall l_{i}=1$ and $a=1$ case, where the time evolution $\mathcal{T}_{1}$ was defined by using the combinatorial $R$. It was extended to general inhomogeneity $l$ and boundary condition $a$ in [HKT2], where it was proved that the $\mathcal{T}_{a}$ is factorized as above into the Weyl group operators. Due to the boundary condition, it can be shown that all the $S_{i}$ act actually as $S_{i}=e_{i}^{\infty}$. The expressions in Definition 1 do not depend on $l$ explicitly. The basic $D_{n}^{(1)}$-automaton corresponding to the situation $\forall l_{i}=1$ will play a fundamental role in the sequel.

\section{Reduction to basic $D_{n}^{(1)}$-automaton}

For the basic $D_{n}^{(1)}$-automaton, the time evolution $\mathcal{T}_{1}$ in Definition 1 was described as a motion of particles and anti-particles that undergo pair creation and annihilation [HKT3]. 
Here we generalize the result to the inhomogeneous $D_{n}^{(1)}$-automaton for any $\mathcal{T}_{a}$. It turns out that the inhomogeneous automaton admits a reduction to the basic one except inserting certain operator $Q$ which will be introduced around (6).

First we prepare some notations for the basic automaton. We identify $B_{1}$ with $J$ as a set via the correspondence $\vec{a} \in B_{1} \leftrightarrow a \in J$. Then states of the basic $D_{n}^{(1)}$-automaton are infinite arrays of the letters from $J$. The totality of them will be denoted by

$$
\widetilde{W}[a]=\left\{\left(\ldots, b_{i}, b_{i+1}, \ldots\right) \in \cdots \times B_{1} \times B_{1} \times \cdots \mid b_{i}=a \text { for }|i| \gg 1\right\} .
$$

In the sequel, we introduce the operators $P, Q, L_{j}$ and $K_{j}$. Except $P$, they are actually dependent on the choice $a$ of the boundary condition. (In appendix B we will write them as $Q^{(a)}$ and $K_{j}^{(a)}$.) For $\vec{x}=\left(x_{1}, \ldots, x_{n}, x_{-n}, \ldots, x_{-1}\right) \in B_{k}$, let $P(\vec{x})$ be an array of $k$ letters arranged as

$$
P(\vec{x})=\overbrace{-1 \ldots-1}^{x_{-1}} \ldots \overbrace{-n \ldots-n}^{x_{-n}} \overbrace{n \ldots n}^{x_{n}} \ldots \overbrace{1 \ldots 1}^{x_{1}} .
$$

For $\boldsymbol{b}=\left(\ldots, \vec{b}_{i}, \vec{b}_{i+1}, \ldots\right) \in W[a]_{l}$, let $P(\boldsymbol{b})$ be an array of infinite letters and walls given by

$$
P(\boldsymbol{b})=\ldots\left|P\left(\vec{b}_{i}\right)\right| P\left(\vec{b}_{i+1}\right) \mid \ldots,
$$

where each $P\left(\vec{b}_{i}\right)$ for $\vec{b}_{i} \in B_{l_{i}}$ is defined by (3). The symbol $\mid$ denotes the wall inserted between every $P\left(\vec{b}_{i}\right)$ and $P\left(\vec{b}_{i+1}\right)$.

Let $\widetilde{W}[a]_{l}$ be the same set as $\widetilde{W}[a]$ except that walls are inserted at the same positions as in the elements of $P\left(W[a]_{l}\right)$. Namely $\widetilde{W}[a]_{l}$ consists of the elements of the form

$$
c=\ldots\left|C_{i}\right| C_{i+1} \mid \ldots,
$$

where $C_{i}(i \in \mathbb{Z})$, which we call a cell, is an array of $l_{i}$ letters from $J$. For distant $i$, i.e., $|i| \gg 1$, the cell $C_{i}$ consists only of the letter $a$. On the other hand $C_{i}$ with not necessarily distant $i$ can assume different ordering from (3), hence $P\left(W[a]_{l}\right) \subset \widetilde{W}[a]_{l}$ holds.

We let $\widetilde{W}[a]_{l}^{Q}$ denote a subset of $\widetilde{W}[a]_{l}$ in which every cell $C_{i}$ has the form

$$
C_{i}=\overbrace{-a \ldots-a}^{s_{i}} \ldots \ldots \overbrace{a \ldots a}^{t_{i}},
$$

involving $\pm a$ nowhere else in the cell $C_{i}$. We let $Q$ be the operator on $\widetilde{W}[a]_{l}^{Q}$ that sends each cell $C_{i}$ in (5) to

$$
C_{i}^{\prime}=\overbrace{a \ldots a}^{t_{i}} \ldots \ldots \overbrace{-a \ldots-a}^{s_{i}},
$$

and keeps the other letters unchanged. By the definition $Q$ becomes trivial for the basic automaton.

For $a \in J$ and $j \in J \backslash\{a,-a\}$ we introduce a map $L_{j}:\left(\mathbb{Z}_{\geq 0}\right) \times B_{1} \rightarrow B_{1} \times\left(\mathbb{Z}_{\geq 0}\right)$ as follows. Let the diagram

$$
m \underset{b^{\prime}}{\stackrel{b}{\longrightarrow}} m^{\prime}
$$

depict the relation $L_{j}:(m, b) \mapsto\left(b^{\prime}, m^{\prime}\right)$. Then under the assumption $m \in \mathbb{Z}_{\geq 0}$ and $b \in B_{1} \backslash\{j,-j, a,-a\}$, the following specify the map $L_{j}$ completely: 


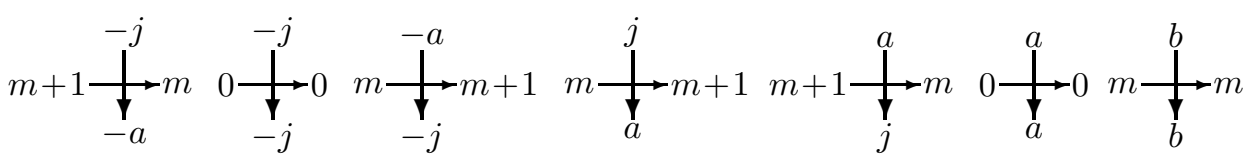

For $j \in J \backslash\{a,-a\}$ we construct an operator $K_{j}: \widetilde{W}[a] \rightarrow \widetilde{W}[a]$ from a composition of $L_{j}$ 's as follows. Given a state of the basic automaton $\left(\ldots, b_{i}, b_{i+1}, \ldots\right) \in \widetilde{W}[a]$, there exists an integer $g$ such that $b_{g^{\prime}}=a$ for all $g^{\prime}<g$ owing to the boundary condition (2). Fix any such $g$. Then the operator $K_{j}$ maps $\left(\ldots, b_{i}, b_{i+1}, \ldots\right)$ to $\left(\ldots, c_{i}, c_{i+1}, \ldots\right)$, where $c_{g^{\prime}}=b_{g^{\prime}}$ for all $g^{\prime}<g$. The remaining $c_{g}, c_{g+1}, \ldots$ are determined by the composition of $L_{j}$ 's as

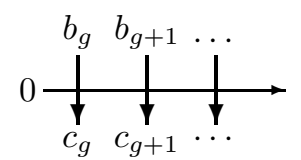

It is easy to see that the result is independent of the choice of $g$. We let $K_{j}$ act also on $\widetilde{W}[a]_{l}$ in the same way as $\widetilde{W}[a]$ by ignoring the walls. For an example if $a=3$, the action of $K_{2}$ on a state $\ldots,-2,2,-3,3,4,-3,-2,3,3, \ldots \in \widetilde{W}[3]$ proceeds as (... denotes the array of 3 only)

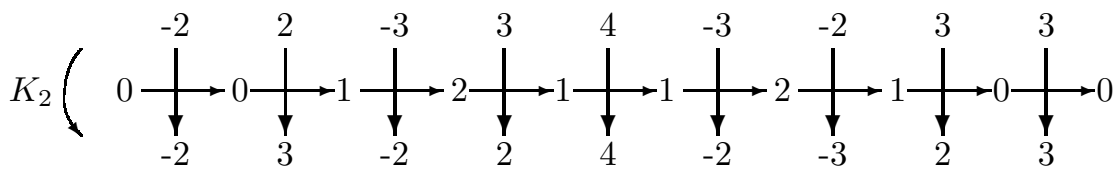

leading to the output $\ldots,-2,3,-2,2,4,-2,-3,2,3, \ldots \in \widetilde{W}[3]$.

Let us present an interpretation as a system of particles and anti-particles with various colors. A state $\left(\ldots, b_{i}, b_{i+1}, \ldots\right) \in \widetilde{W}[a]$ is regarded as a one dimensional array of boxes. The local state $b_{i}=b$ with $b \in B_{1} \backslash\{a,-a\}$ means that the $i$-th box contains a particle of color $b$. Since the set $B_{1} \backslash\{a,-a\}$ is invariant under the interchange $b \leftrightarrow-b$, the local state $-b$ may be viewed as a box containing an anti-particle of $b$ and vice versa. We regard the selected local state $a \in B_{1}$ as an empty box. On the other hand, when acting $K_{j}$ on $\widetilde{W}[a]$, the local state $-a \in B_{1}$ plays a special role as a bound state of a particle of color $j$ and its anti-particle $-j$. The boundary condition in (2) allows only finitely many boxes to be non-empty. Under this interpretation, the operator $K_{j}$ describes the dynamics of right-moving particles of color $j$ seeking the empty box $a$ or a free partner, i.e., an anti-particle $-j$ not yet paired with the other $j$ 's, to form a bound state $-a$ within a box. To see this, regard the diagram (7) as representing $m$ incident particles with color $j$ coming from the left and $m^{\prime}$ particles with the same color $j$ outgoing to the right. The propagation of the color $j$ particles to the right thereby induces the change of contents in a box corresponding to the transformation $b \rightarrow b^{\prime}$. In fact, in the first figure of (8), a color $j$ particles coming from the left is trapped in a box to form the bound state $-a$ with the anti-particle $-j$ (pair annihilation $(j,-j) \rightarrow-a$ ). In the second figure, there are no color $j$ particles coming from the left nor in the box hence nothing happens. In the third figure, a color $j$ particle is removed off the bound state $-a$ leaving the anti-particle $-j$ (pair creation $-a \rightarrow(j,-j)$ ). In the fourth figure, a color $j$ particle 
is taken away to the right leaving an empty box $a$. In the fifth figure, a color $j$ particle stops at the empty box to fill it. In the last two figures, nothing can happen. When $K_{j}$ acts on $\widetilde{W}[a]_{l}$ instead of $\widetilde{W}[a]$, it induces the same motion of particles without any effect

from the walls. In contrast with this, the operator $Q$ remembers the walls at $l=\left(l_{i}\right)_{i \in \mathbb{Z}}$ and swaps the positions of the empty boxes $a$ and the bound states $-a$ within each cell.

Definition 3. For $1 \leq a \leq n$ we define the operator $T_{a}$ on $W[a]_{l}$ as

$$
T_{a}=P^{-1}\left(\prod_{j=a+1}^{n} K_{j}\right)\left(\prod_{j=-n}^{-(a+1)} K_{j}\right)\left(\prod_{j=-(a-1)}^{-1} K_{j}\right) Q\left(\prod_{j=1}^{a-1} K_{j}\right) P
$$

and $T_{-a}$ on $W[-a]_{l}$ as

$$
T_{-a}=P^{-1}\left(\prod_{j=-(a-1)}^{-1} K_{j}\right) Q\left(\prod_{j=1}^{a-1} K_{j}\right)\left(\prod_{j=a+1}^{n} K_{j}\right)\left(\prod_{j=-n}^{-(a+1)} K_{j}\right) P,
$$

where $\prod_{j=c}^{c-1} K_{j}=i d$, and for $c \leq d$ we adopt the convention $\prod_{j=c}^{d} K_{j}=K_{c} K_{c+1} \cdots K_{d}$.

One should remember that the operators $K_{j}$ and $Q$ (and $L_{j}$ ) are actually dependent on $a$ as opposed to $P$. Up to this distinction, the expressions of $T_{n}$ and $T_{-n}$ look formally identical. See Remark 20 for the possible rearrangement of these products.

Example 4. The time evolution operators for inhomogeneous $D_{4}^{(1)}$-automaton read as

$$
\begin{aligned}
& T_{1}=P^{-1} K_{2} K_{3} K_{4} K_{-4} K_{-3} K_{-2} Q P, \\
& T_{2}=P^{-1} K_{3} K_{4} K_{-4} K_{-3} K_{-1} Q K_{1} P, \\
& T_{3}=P^{-1} K_{4} K_{-4} K_{-2} K_{-1} Q K_{1} K_{2} P, \\
& T_{4}=P^{-1} K_{-3} K_{-2} K_{-1} Q K_{1} K_{2} K_{3} P \\
& T_{-4}=P^{-1} K_{-3} K_{-2} K_{-1} Q K_{1} K_{2} K_{3} P, \\
& T_{-3}=P^{-1} K_{-2} K_{-1} Q K_{1} K_{2} K_{4} K_{-4} P, \\
& T_{-2}=P^{-1} K_{-1} Q K_{1} K_{3} K_{4} K_{-4} K_{-3} P, \\
& T_{-1}=P^{-1} Q K_{2} K_{3} K_{4} K_{-4} K_{-3} K_{-2} P .
\end{aligned}
$$

Each operator $K_{j}(j \in J)$ in Definition 3 transforms the cells as

$$
\ldots \overbrace{a \ldots a}^{\alpha} \overbrace{j \ldots j}^{\beta} \ldots \overbrace{-j \ldots-j}^{\gamma} \overbrace{-a \ldots-a}^{\delta} \ldots \mapsto \ldots \overbrace{j \ldots j}^{\alpha^{\prime}} \overbrace{a \ldots a}^{\beta^{\prime}} \ldots \overbrace{-a \ldots-a}^{\gamma^{\prime}} \overbrace{-j \ldots-j}^{\delta^{\prime}} \ldots,
$$

for $-n \leq j \leq-1$, and as

$$
\ldots \overbrace{-j \ldots-j}^{\gamma} \overbrace{-a \ldots-a}^{\delta} \ldots \overbrace{a \ldots a}^{\alpha} \overbrace{j \ldots j}^{\beta} \ldots \mapsto \ldots \overbrace{-a \ldots-a}^{\gamma^{\prime}} \overbrace{-j \ldots-j}^{\delta^{\prime}} \ldots \overbrace{j \ldots j}^{\alpha^{\prime}} \overbrace{a \ldots a}^{\beta^{\prime}} \ldots,
$$

for $1 \leq j \leq n$, where $\alpha+\beta=\alpha^{\prime}+\beta^{\prime}$ and $\gamma+\delta=\gamma^{\prime}+\delta^{\prime}$. The letters other than $\pm j$ and $\pm a$ are kept unchanged. In (12) and (13), the ordering of the letters are the same as (3) except the positions of $\pm a$. See Example 14. This fact assures that the right hand sides of (10) and (11) without the leftmost $P^{-1}$ belong to $P\left(W[a]_{l}\right)$, hence Definition 3 makes sense. 
Example 5. Consider $T_{-4}$ for $D_{4}^{(1)}$ given in Example 4. The operators $K_{j}$ and $Q$ therein act as (to save the space we write $\overline{4}$ to mean -4 etc.)

$$
\begin{array}{ll} 
& \ldots|\overline{3} 431| \overline{1} \overline{2}|\overline{4} 2| \overline{4} 31|\overline{4} \overline{4} \overline{4}| \ldots \\
\stackrel{K_{3}}{\longmapsto} & \ldots|\overline{3} \overline{3} \overline{4} 1| \overline{1} \overline{2}|32| 3 \overline{4} 1|3 \overline{4} \overline{4}| \ldots \\
\stackrel{K_{2}}{\longmapsto} & \ldots|\overline{3} \overline{3} \overline{4} 1| \overline{1} \overline{2}|3 \overline{4}| 321|3 \overline{4} \overline{4}| \ldots \\
\stackrel{K_{1}}{\longmapsto} & \ldots|\overline{3} \overline{3} \overline{4} \overline{4}| 4 \overline{2}|3 \overline{4}| 32 \overline{4}|31 \overline{4}| \ldots \\
\stackrel{Q}{\longmapsto} & \ldots|\overline{4} \overline{4} \overline{3} \overline{3}| \overline{2} 4|\overline{4} 3| \overline{4} 32|\overline{4} 31| \ldots \\
\stackrel{K_{-1}}{\longmapsto} & \ldots|\overline{4} \overline{4} \overline{3} \overline{3}| \overline{2} 1|\overline{1} 3| \overline{4} 32|\overline{4} 31| \ldots \\
\stackrel{K_{-2}}{\longmapsto} & \ldots|\overline{4} \overline{4} \overline{4} \overline{3}| \overline{4} 1|\overline{1} 3| \overline{2} 32|\overline{4} 31| \ldots \\
\stackrel{K_{-3}}{\longmapsto} & \ldots|\overline{4} \overline{4} \overline{4} \overline{4}| \overline{3} 1|\overline{1} 4| \overline{2} 32|\overline{4} 31| \ldots .
\end{array}
$$

where ... stands for the cells that consist of $\overline{4}$ only. Both the initial and the final states belong to $P\left(W[-4]_{l}\right)$ with $l=(\ldots, 4,2,2,3,3, \ldots)$.

For $a \neq \pm n$, the time evolution $T_{a}$ involves the factor $K_{n} K_{-n}$. At the intermediate state between $K_{n}$ and $K_{-n}$, the cells can contain the letters $n$ and $-n$ simultaneously, but $a$ and $-a$ can not coexist. For the other intermediate states, the situation is opposite, namely, $a$ and $-a$ can coexist but $n$ and $-n$ can not. The following is such an example. See also Example 14.

Example 6. Consider $T_{1}$ for $D_{4}^{(1)}$ given in Example 4. The operators $K_{j}$ and $Q$ therein act as (to save the space we write $\overline{4}$ to mean -4 etc.)

$$
\begin{array}{ll} 
& \ldots|\overline{4} \overline{4}| \overline{3} 431|\overline{1} \overline{3} \overline{4}| \overline{2}|\overline{1} 1| 111 \mid \ldots \\
\stackrel{Q}{\longmapsto} & \ldots|\overline{4} \overline{4}| 1 \overline{3} 43|\overline{3} \overline{4} \overline{1}| \overline{2}|1 \overline{1}| 111 \mid \ldots \\
\stackrel{K_{-2}}{\longmapsto} & \ldots|\overline{4} \overline{4}| 1 \overline{3} 43|\overline{3} \overline{4} 2| 1|\overline{2} 2| \overline{2} \overline{2} 1 \mid \ldots \\
\stackrel{K_{-3}}{\longmapsto} & \ldots|\overline{4} \overline{4}| 114 \overline{1}|1 \overline{4} 2| \overline{3}|\overline{2} 2| \overline{2} \overline{2} 1 \mid \ldots \\
\stackrel{K_{-4}}{\longmapsto} & \ldots|11| \overline{4} \overline{4} 44|\overline{4} 12| \overline{3}|\overline{2} 2| \overline{2} \overline{2} \overline{4} \mid \ldots \\
\stackrel{K_{4}}{\longmapsto} & \ldots|11| \overline{4} \overline{4} 11|\overline{1} 42| \overline{3}|\overline{2} 2| \overline{2} \overline{2} \overline{4} \mid \ldots \\
\stackrel{K_{3}}{\longmapsto} & \ldots|11| \overline{4} \overline{4} 11|\overline{3} 42| \overline{1}|\overline{2} 2| \overline{2} \overline{2} \overline{4} \mid \ldots \\
\stackrel{K_{2}}{\longmapsto} & \ldots|11| \overline{4} \overline{4} 11|\overline{3} 41| \overline{2}|\overline{1} 1| \overline{1} \overline{1} \overline{4} \mid \ldots
\end{array}
$$

where ... stands for the cells that consist of 1 only. Both the initial and the final states belong to $P\left(W[1]_{l}\right)$ with $l=(\ldots, 2,4,3,1,2,3, \ldots)$.

Now we give the main theorem of this paper.

Theorem 7. For any $a \in J, \mathcal{T}_{a}=T_{a}$ is valid. Namely, the time evolution operator $\mathcal{T}_{a}$ in Definition 1 coincides with $T_{a}$ in Definition 3 that admits the particle interpretation.

We include a proof in section 5 and appendix B. 
Table 1: Sets $B_{k}=\{\vec{x} \mid(*)\}$

\begin{tabular}{c|c|c} 
algebra & array $\vec{x}$ & condition $(*)$ \\
\hline$A_{2 n-1}^{(2)}$ & $\left(x_{1}, \ldots, x_{n}, x_{-n}, \ldots, x_{-1}\right) \in \mathbb{Z}_{\geq 0}^{2 n}$ & $s(\vec{x})=k$ \\
$A_{2 n}^{(2)}$ & $\left(x_{1}, \ldots, x_{n}, x_{-n}, \ldots, x_{-1}\right) \in \mathbb{Z}_{\geq 0}^{2 n}$ & $s(\vec{x}) \leq k$ \\
$B_{n}^{(1)}$ & $\left(x_{1}, \ldots, x_{n}, x_{0}, x_{-n}, \ldots, x_{-1}\right) \in \mathbb{Z}_{\geq 0}^{2 n+1}$ & $x_{0} \in\{0,1\}, s(\vec{x})=k$ \\
$C_{n}^{(1)}$ & $\left(x_{1}, \ldots, x_{n}, x_{-n}, \ldots, x_{-1}\right) \in \mathbb{Z}_{\geq 0}^{2 n}$ & $s(\vec{x}) \in k-2 \mathbb{Z}_{\geq 0}$ \\
$D_{n}^{(1)}$ & $\left(x_{1}, \ldots, x_{n}, x_{-n}, \ldots, x_{-1}\right) \in \mathbb{Z}_{\geq 0}^{2 n}$ & $x_{n} x_{-n}=0, s(\vec{x})=k$ \\
$D_{n+1}^{(2)}$ & $\left(x_{1}, \ldots, x_{n}, x_{0}, x_{-n}, \ldots, x_{-1}\right) \in \mathbb{Z}_{\geq 0}^{2 n+1}$ & $x_{0} \in\{0,1\}, s(\vec{x}) \leq k$
\end{tabular}

\section{Embedding into inhomogeneous $D_{n}^{(1)}$-automaton}

In this section an automaton means an inhomogeneous automaton. The $D_{n}^{(1)}$-automaton $(n \geq 4)$ contains, as certain sectors, all the automata associated with the Kang-KashiwaraMisra (KKM) crystals $[\mathrm{KKM}]$ for the other non-exceptional affine Lie algebras $A_{2 n-3}^{(2)}$, $A_{2 n-4}^{(2)}, B_{n-1}^{(1)}, C_{n-2}^{(1)}$ and $D_{n-1}^{(2)}$. Here we describe the embedding of these automata along the following scheme:

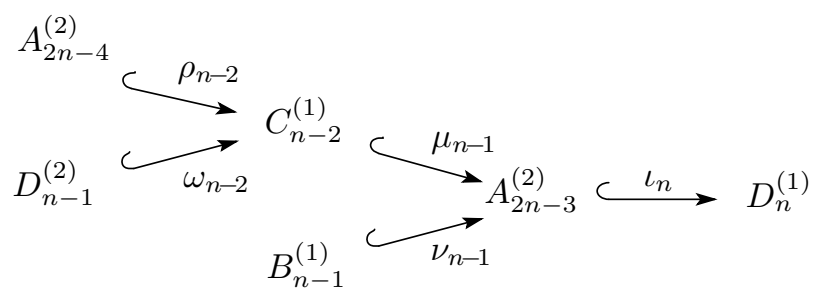

By embedding of an automaton, we precisely mean the following. Take any pair $\psi$ : $X \hookrightarrow X^{\prime}$ in the above scheme. Then there is an injection $\psi: B_{k} \rightarrow B_{k^{\prime}}^{\prime}$ between the KKM crystals associated with these algebras $X$ and $X^{\prime}$, respectively. Moreover, this $\psi$ has an extension to Kashiwara operators $[\mathrm{K}] e_{i}, f_{i}$ in such a way that $\psi\left(e_{i} \vec{x}\right)=\psi\left(e_{i}\right) \psi(\vec{x})$ and $\psi\left(f_{i} \vec{x}\right)=\psi\left(f_{i}\right) \psi(\vec{x})$ for any $\vec{x} \in B_{k}$ and $i$. It follows that the combinatorial $R$ for $X$ is embedded into the one for $X^{\prime}$ as $R=\left(\psi^{-1} \otimes \psi^{-1}\right) R^{\prime}(\psi \otimes \psi)$. The map $\psi^{\Delta}=$ $(\cdots \otimes \psi \otimes \psi \otimes \cdots)$ embedds the states of the $X$-automaton into $X^{\prime}$-automaton and relates their time evolutions simply by $T_{a}^{X}=\left(\psi^{\Delta}\right)^{-1} T_{a^{\prime}}^{X^{\prime}} \psi^{\Delta}$. The precise correspondence of the boundary conditions $a$ and $a^{\prime}$ will be described below together with the injections $\iota_{n}, \mu_{n}, \nu_{n}, \rho_{n}$ and $\omega_{n}$. The embedding $\rho_{n}$ and $\omega_{n}$ for the basic automata have appeared in [HKOTY]. The $\mu_{n}$ and $\iota_{n}$ have been introduced in section 6.1 of [KOTY2] in a tropical setting.

In Table 1, the KKM crystal $B_{k}$ is listed for each algebra as a set. They all have the presentation $B_{k}=\left\{\vec{x}=\left(x_{1}, \ldots, x_{-1}\right) \in \mathbb{Z}_{>0}^{N} \mid(*)\right\}$ with certain $N$ and the condition $(*)$ on $\vec{x}$. We let $s(\vec{x})=x_{1}+\cdots+x_{-1}$ denote the sum of all the components of $\vec{x}$ and list the array $\vec{x}$ and $(*)$. The images of the maps appearing in the embedding scheme are given in Table 2. The component $x_{0}$ appearing in the image $\mu_{n}(\vec{x})$ is the non-negative integer 
Table 2: Maps $\iota_{n}, \mu_{n}, \nu_{n}, \rho_{n}, \omega_{n}$

\begin{tabular}{c|c|c}
$\psi$ & $\psi(\vec{x})$ & $\psi\left(e_{i}\right)$ \\
\hline$\iota_{n}: B_{k} \rightarrow B_{k}^{\prime}$ & $\left(x_{1}, \ldots, x_{n-1}, 0,0, x_{-n+1}, \ldots, x_{-1}\right)$ & $e_{i}$ \\
$\mu_{n}: B_{k} \rightarrow B_{k}^{\prime}$ & $\left(x_{0}, x_{1}, \ldots, x_{n-1}, x_{-n+1}, \ldots, x_{-1}, x_{0}\right)$ & $e_{i+1}$ \\
$\nu_{n}: B_{k} \rightarrow B_{2 k}^{\prime}$ & $\left(2 x_{1}, \ldots, 2 x_{n-1}, 2 x_{n}+x_{0}, 2 x_{-n}+x_{0}, 2 x_{-n+1}, \ldots, 2 x_{-1}\right)$ & $e_{i}^{2-\delta_{i, n}}$ \\
$\rho_{n}: B_{k} \rightarrow B_{2 k}^{\prime}$ & $\left(2 x_{1}, \ldots, 2 x_{n}, 2 x_{-n}, \ldots, 2 x_{-1}\right)$ & $e_{i}^{2-\delta_{i, 0}}$ \\
$\omega_{n}: B_{k} \rightarrow B_{2 k}^{\prime}$ & $\left(2 x_{1}, \ldots, 2 x_{n-1}, 2 x_{n}+x_{0}, 2 x_{-n}+x_{0}, 2 x_{-n+1}, \ldots, 2 x_{-1}\right)$ & $e_{i}^{2-\delta_{i, 0}-\delta_{i, n}}$
\end{tabular}

specified by $x_{0}=\left(k-\sum_{j=1}^{n-1}\left(x_{j}+x_{-j}\right)\right) / 2$. The image $\psi\left(f_{i}\right)$ is obtained from $\psi\left(e_{i}\right)$ by changing the letter $e$ to $f$.

As an example let us illustrate the embedding $\iota_{n}$ in further detail. This is a significant case in that the $A_{2 n-3}^{(2)}$-automaton is considerably simpler than the $D_{n}^{(1)}$-automaton and all the other automata can firstly be embedded into the former.

In view of $\iota_{n}:\left(x_{1}, \ldots, x_{n-1}, x_{-n+1}, \ldots, x_{-1}\right) \mapsto\left(x_{1}, \ldots, x_{n-1}, 0,0, x_{-n+1}, \ldots, x_{-1}\right)$, the $A_{2 n-3}^{(2)}$-automaton is a restriction of the $D_{n}^{(1)}$-automaton to those local states satisfying $x_{n}=x_{-n}=0$. The set of states is formally the same as (1) once $B_{l_{i}}$ is understood as the one for $A_{2 n-3}^{(2)}$. The boundary condition is specified by a letter $a \in\{ \pm 1, \ldots, \pm(n-1)\}$. The time evolutions $T_{a}$ for these $a$ are still given by the formulas in Definition 3 except only that the states they act are restricted. The $T_{a}$ with $a \neq \pm n$ contains the operators $K_{n}$ and $K_{-n}$ only through the combination $K_{n} K_{-n}$. See Example 4 for $n=4$. In Lemma 21 we will show that the composition $K_{n} K_{-n}$ under the restriction $x_{n}=x_{-n}=$ 0 coincides with a simple operator $K_{-a}$ defined as follows. (Note that $K_{-a}$ was not introduced in $D_{n}^{(1)}$ case.) The $K_{-a}$ is the composition of $L_{-a}$ in the same was as (9), and the $L_{-a}$ is specified by the diagrams:

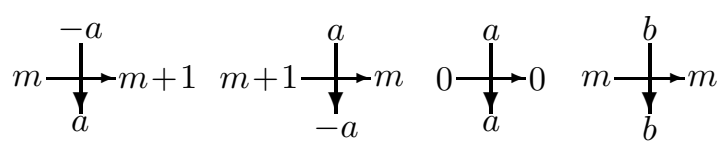

where $b \in B \backslash\{a,-a\}$ and $m \in \mathbb{Z}_{\geq 0}$. These diagrams represent the motion of the rightmoving particles $-a$ seeking the empty box $a$ without pair creation nor annihilation. Such a behavior of color $-a$ particles is exceptional, in that they still act as bound states of $j$ and $-j$ under the operator $K_{j}$ with $j \neq-a$.

Example 8. The time evolution operators for $A_{5}^{(2)}$-automaton read as

$$
\begin{aligned}
& T_{1}=P^{-1} K_{2} K_{3} K_{-1} K_{-3} K_{-2} Q P, \\
& T_{2}=P^{-1} K_{3} K_{-2} K_{-3} K_{-1} Q K_{1} P, \\
& T_{3}=P^{-1} K_{-3} K_{-2} K_{-1} Q K_{1} K_{2} P, \\
& T_{-3}=P^{-1} K_{-2} K_{-1} Q K_{1} K_{2} K_{3} P, \\
& T_{-2}=P^{-1} K_{-1} Q K_{1} K_{3} K_{2} K_{-3} P, \\
& T_{-1}=P^{-1} Q K_{2} K_{3} K_{1} K_{-3} K_{-2} P .
\end{aligned}
$$


Example 9. Consider $T_{3}$ for $A_{5}^{(2)}$ given in Example 8. The operators $K_{j}$ and $Q$ therein act as (to save the space we write $\overline{3}$ to mean -3 etc.)

$$
\begin{array}{ll} 
& \ldots|11| \overline{1} \overline{1} \overline{3} 1|\overline{1} 32| 3333 \mid \ldots \\
\stackrel{K_{2}}{\longmapsto} & \ldots|11| \overline{1} \overline{1} \overline{2} 1|\overline{1} 23| 2333 \mid \ldots \\
\stackrel{K_{1}}{\longmapsto} & \ldots|33| \overline{3} \overline{3} \overline{2} 3|\overline{3} 23| 2333 \mid \ldots \\
\stackrel{Q}{\longmapsto} & \ldots|33| 3 \overline{2} \overline{3} \overline{3}|32 \overline{3}| 3332 \mid \ldots \\
\stackrel{K_{-1}}{\longmapsto} & \ldots|33| 3 \overline{2} 11|\overline{1} 21| \overline{1} \overline{1} 32 \mid \ldots \\
\stackrel{K_{-2}}{\longmapsto} & \ldots|33| 3311|\overline{1} \overline{3} 1| \overline{1} \overline{1} 32 \mid \ldots \\
\stackrel{K_{-3}}{\longmapsto} & \ldots|33| 3311|\overline{1} 31| \overline{1} \overline{1} \overline{3} 2 \mid \ldots
\end{array}
$$

where... stands for the cells that consist of 3 only.

Let us briefly comment on the $A_{n-1}^{(1)}$ case where the associated automaton is known as the (generalized) box-ball system [TS, T, HHIKTT, FOY]. The relevant crystal $B_{k}$ is the set $\left\{\vec{x}=\left(x_{1}, \ldots, x_{n}\right) \in \mathbb{Z}_{>0}^{n} \mid x_{1}+\cdots+x_{n}=k\right\}$ equipped with the functions $\varphi_{i}(\vec{x})=x_{i}, \varepsilon_{i}(\vec{x})=x_{i+1}$ and the Kashiwara operators $e_{i}(\vec{x})=\left(\ldots, x_{i}+1, x_{i+1}-1, \ldots\right)$, $f_{i}(\vec{x})=\left(\ldots, x_{i}-1, x_{i+1}+1, \ldots\right)$, where all the indices are considered to be in $\mathbb{Z} / n \mathbb{Z}$. We set $J=\{1,2, \ldots, n\}$ and $\vec{a}=\left(x_{i}=k \delta_{i, a}\right) \in B_{k}$ for $a \in J$. In particular $\vec{a} \in B_{1}$ will simply be called $a$. Under these notations the automaton states are again specified by (1). Due to the Dynkin diagram $\mathbb{Z}_{n}$ symmetry, there is no loss of generality to restrict ourselves to the case $a=1$. Then the time evolution is given by the Weyl group operators as $\mathcal{T}_{1}=\sigma^{\Delta} S_{2} S_{3} \cdots S_{n-1} S_{0}$ [HKT2], where $\sigma^{\Delta}=\cdots \otimes \sigma \otimes \sigma \otimes \cdots$ is defined by $\sigma\left(\left(x_{1}, x_{2}, \ldots, x_{n}\right)\right)=\left(x_{2}, \ldots, x_{n}, x_{1}\right)$. In the same way as section 5 and appendix B, we have proved that $\mathcal{T}_{1}=T_{1}$ holds, where $T_{1}$ consists of the particle motion operator as

$$
T_{1}=P^{-1} K_{2} K_{3} \cdots K_{n} Q P .
$$

Here $P$ acts on $W[1]_{l}$ as (4) with (3) simplified as

$$
P(\vec{x})=\overbrace{n \ldots n}^{x_{n}} \ldots \overbrace{2 \ldots 2}^{x_{2}} \overbrace{1 \ldots 1}^{x_{1}} .
$$

The operator $Q$ rearranges each cell (16) into $\overbrace{1 \ldots 1}^{x_{1}} \overbrace{n \ldots n}^{x_{n}} \ldots \overbrace{2 \ldots 2}^{x_{2}}$. The operator $K_{j}$ is defined in terms of the vertex diagrams $(2 \leq j \leq n, b \neq 1, j)$ :

$$
m \underset{1}{\stackrel{f}{\longrightarrow}} m+1 \quad m+1 \underset{j}{\stackrel{f}{\longrightarrow}} m \quad 0 \underset{1}{\stackrel{1}{\longrightarrow}} 0 \quad m \underset{b}{\stackrel{b}{\longrightarrow}} m
$$

These rules coincide with $T_{1}$ of the $D_{n}^{(1)}$-automaton restricted to the sector without antiparticles, i.e., those local states $\left(x_{1}, \ldots, x_{n}, x_{-n}, \ldots, x_{-1}\right)$ such that $x_{-n}=\cdots x_{-1}=0$. In fact, the absence of anti-particles reduces the diagrams (8) to the last four, which yield (17) under the choice $a=1$. Thus we conclude that the inhomogeneous box-ball system is reduced to the basic case via (15). Moreover, it coincides with a sector of the $D_{n}^{(1)}$ automaton without anti-particles. The result equivalent to (15) has also been obtained in eq.(10) of [HHIKTT] and by purely combinatorial arguments in $[\mathrm{F}]$. The diagrams equivalent to (17) appeared in [HIK] for the basic box-ball system. 


\section{$5 \quad$ Weyl group operator $S_{i}$ as particle motion $K_{j}$}

Our main theorem $\mathcal{T}_{a}=T_{a}$ for $D_{n}^{(1)}$ is a composition of finer relations that intertwine the Weyl group operators $S_{i}$ and the particle motion operators $K_{j}$. The aim of this section is to present them in Proposition 11. Many assertions and formulas provided below for general case will be recognized most easily by consulting $n=4$ case in Examples 13, 14 as well as Examples 2 and 4.

For $\vec{x}=\left(x_{1}, \ldots, x_{n}, x_{-n}, \ldots, x_{-1}\right) \in B_{k}$, let $\bar{P}(\vec{x})$ be an array of $k$ letters arranged as

$$
\bar{P}(\vec{x})=\overbrace{1 \ldots 1}^{x_{1}} \overbrace{-2 \ldots-2}^{x_{-2}} \ldots \overbrace{-n \ldots-n}^{x_{-n} \ldots \ldots n} \overbrace{n \ldots}^{x_{n}} \ldots \overbrace{2 \ldots 2}^{x_{2}} \overbrace{-1 \ldots-1}^{x_{-1}},
$$

which is obtained from $P(\vec{x})$ in (3) by interchanging the positions of 1 and -1 . Similalry to (4), we set $\bar{P}(\boldsymbol{b})=\ldots\left|\bar{P}\left(\vec{b}_{i}\right)\right| \bar{P}\left(\vec{b}_{i+1}\right) \mid \ldots$ for $\boldsymbol{b}=\left(\ldots, \vec{b}_{i}, \vec{b}_{i+1}, \ldots\right) \in W[a]_{l}$.

In section 2 we introduced the Weyl group operator $S_{j}$ acting on $\coprod_{a \in J} W[a]_{l}$. Here we use two further kinds of Weyl group operators $r_{j}, s_{j}(0 \leq j \leq n)$. The $r_{j}: B_{1} \rightarrow B_{1}$ is the one for the crystal $B_{1}$. In the crystal graph depicted in Figure 1, if there is a color $j$ arrow coming into or outgoing from the node $p$, the image $r_{j}(p)$ is determined by the relation $r_{j}(p) \stackrel{j}{\rightarrow} p$ or $r_{j}(p) \stackrel{j}{\leftarrow} p$, respectively. If there is no such arrow, $r_{j}(p)=p$. The $s_{j}$ is the one that acts on $\coprod_{a \in J} \widetilde{W}[a]_{l}$ totally componentwise ignoring the walls. Namely, it transforms the cells as

$$
\begin{aligned}
\boldsymbol{c} & =\ldots\left|C_{i}\right| C_{i+1} \mid \ldots, & C_{i} & =p_{1} \ldots p_{l_{i}} \in B_{1}^{\times l_{i}}, \\
s_{j}(\boldsymbol{c}) & =\ldots\left|C_{i}^{\prime}\right| C_{i+1}^{\prime} \mid \ldots, & C_{i}^{\prime} & =r_{j}\left(p_{1}\right) \ldots r_{j}\left(p_{l_{i}}\right) \in B_{1}^{\times l_{i}} .
\end{aligned}
$$

Obviously, one has

$$
S_{i}\left(W[a]_{l}\right) \subseteq W\left[r_{i}(a)\right]_{l}, \quad s_{i}\left(\widetilde{W}[a]_{l}\right) \subseteq \widetilde{W}\left[r_{i}(a)\right]_{l} .
$$

For $a \in J$ let

$$
\mathcal{T}_{a}=\sigma^{\Delta} S_{i_{2 n-2}} \cdots S_{i_{1}}
$$

be the expression of $\mathcal{T}_{a}$ given in Definition 1 . The sequence $i_{1}, i_{2}, \ldots, i_{2 n-2}$ has the property

$$
\varepsilon_{i_{t}}\left(r_{i_{t-1}} \cdots r_{i_{2}} r_{i_{1}}(a)\right)>0, \quad 1 \leq t \leq 2 n-2,
$$

where $\varepsilon_{i}$ is defined in (37). The operator $T_{a}$ in Definition 3 is expressed as

$$
\begin{aligned}
& T_{a}=P^{-1} Q^{\delta_{a,-1}} K_{j_{2 n-2}}^{\prime} \cdots K_{j_{1}}^{\prime} P, \\
& K_{j}^{\prime} \in\left\{K_{j}, K_{j} Q\right\},
\end{aligned}
$$

with the sequence $j_{1}, j_{2}, \ldots j_{2 n-2} \in J$ specified by

$$
j_{t}=r_{i_{1}} r_{i_{2}} \cdots r_{i_{t-1}} r_{i_{t}} r_{i_{t-1}} \cdots r_{i_{2}} r_{i_{1}}(a), \quad 1 \leq t \leq 2 n-2 .
$$

In view of (19), the operator $S_{i_{t}}$ in (20) acts among the sets:

$$
S_{i_{t}}: W\left[r_{i_{t-1}} \cdots r_{i_{1}}(a)\right]_{l} \rightarrow W\left[r_{i_{t}} \cdots r_{i_{1}}(a)\right]_{l}
$$

for $a \in J$ and $1 \leq t \leq 2 n-2$.

Let $r: B_{1} \rightarrow B_{1}$ be the involutive automorphism $1 \leftrightarrow-1$ and $n \leftrightarrow-n$ keeping the other letters unchanged. It is easy to check that $r_{i_{1}} \cdots r_{i_{2 n-2}}=r$ for all $a \in J$. Consequently $s\left(=s^{-1}\right)=s_{i_{1}} \cdots s_{i_{2 n-2}}: \coprod_{a \in J} \widetilde{W}[a]_{l} \rightarrow \coprod_{a \in J} \widetilde{W}[a]_{l}$ is also independent of $a$, and induces $r$ componentwise. 


\section{Lemma 10.}

$$
\begin{aligned}
& \sigma^{\Delta} \bar{P}^{-1} s=P^{-1} \quad \text { on } P\left(\coprod_{b \in J} W[b]_{l}\right), \\
& \sigma^{\Delta} P^{-1} s=P^{-1} Q \quad \text { on } \bar{P}\left(\coprod_{b \in J} W[b]_{l}\right),
\end{aligned}
$$

where $Q$ is the one for $a=-1$ case in (5)-(6).

The essential task in proving Theorem 7 is to establish

Proposition 11. For any $a \in J$ and $1 \leq t \leq 2 n-2$, the action of the Weyl group operator $S_{i_{t}}$ in (24) is expressed as follows:

$$
\begin{aligned}
& S_{i_{t}}=P^{-1} s_{i_{t}} \cdots s_{i_{1}} K_{j_{t}} s_{i_{1}} \cdots s_{i_{t-1}} P \quad \text { if } K_{j_{t}}^{\prime}, \cdots, K_{j_{1}}^{\prime} \text { do not contain } Q, \\
& =\bar{P}^{-1} s_{i_{t}} \cdots s_{i_{1}} K_{j_{t}} Q s_{i_{1}} \cdots s_{i_{t-1}} P \quad \text { if } K_{j_{t}}^{\prime}=K_{j_{t}} Q \text {, } \\
& =\bar{P}^{-1} s_{i_{t}} \cdots s_{i_{1}} K_{j_{t}} s_{i_{1}} \cdots s_{i_{t-1}} \bar{P} \quad \text { otherwise. }
\end{aligned}
$$

In fact, substituting (27)-(29) into (20) and reducing the result by Lemma 10, $s_{i}^{2}=i d$ and $s_{i_{2 n-2}} \cdots s_{i_{1}}=s$, one gets (22). Note that $a=-1$ is the unique case where (28) and (29) become irrelevant for all $1 \leq t \leq 2 n-2$. Accordingly the relation (26) is used only for $a=-1$ and the other cases are dealt with (25). A proof of Proposition 11 is included in Appendix B.

Remark 12. The case (28) is relevant to $S_{0}$ only. The operator $S_{1}$ is always expressible by $(27)$.

Example 13. For $D_{4}^{(1)}$, formulas (27)-(29) read

$$
\begin{aligned}
& a=1 \quad a=2 \\
& S_{0}=\bar{P}^{-1} s_{0} K_{-2} Q P, \quad S_{1}=P^{-1} s_{1} K_{1} P \\
& S_{2}=\bar{P}^{-1} s_{2} s_{0} K_{-3} s_{0} \bar{P}, \quad S_{0}=\bar{P}^{-1} s_{0} s_{1} K_{-1} Q s_{1} P, \\
& S_{3}=\bar{P}^{-1} s_{3} s_{2} s_{0} K_{-4} s_{0} s_{2} \bar{P}, \quad S_{2}=\bar{P}^{-1} s_{2} s_{0} s_{1} K_{-3} s_{1} s_{0} \bar{P}, \\
& S_{4}=\bar{P}^{-1} s_{4} s_{3} s_{2} s_{0} K_{4} s_{0} s_{2} s_{3} \bar{P}, \quad S_{3}=\bar{P}^{-1} s_{3} s_{2} s_{0} s_{1} K_{-4} s_{1} s_{0} s_{2} \bar{P}, \\
& S_{2}=\bar{P}^{-1} s_{2} s_{4} s_{3} s_{2} s_{0} K_{3} s_{0} s_{2} s_{3} s_{4} \bar{P}, \quad S_{4}=\bar{P}^{-1} s_{4} s_{3} s_{2} s_{0} s_{1} K_{4} s_{1} s_{0} s_{2} s_{3} \bar{P}, \\
& S_{0}=\bar{P}^{-1} s_{0} s_{2} s_{4} s_{3} s_{2} s_{0} K_{2} s_{0} s_{2} s_{3} s_{4} s_{2} \bar{P}, \quad S_{2}=\bar{P}^{-1} s_{2} s_{4} s_{3} s_{2} s_{0} s_{1} K_{3} s_{1} s_{0} s_{2} s_{3} s_{4} \bar{P}, \\
& a=3 \quad a=4 \\
& S_{2}=P^{-1} s_{2} K_{2} P, \quad S_{3}=P^{-1} s_{3} K_{3} P \\
& S_{1}=P^{-1} s_{1} s_{2} K_{1} s_{2} P, \quad S_{2}=P^{-1} s_{2} s_{3} K_{2} s_{3} P, \\
& S_{0}=\bar{P}^{-1} s_{0} s_{1} s_{2} K_{-1} Q s_{2} s_{1} P, \quad S_{1}=P^{-1} s_{1} s_{2} s_{3} K_{1} s_{3} s_{2} P, \\
& S_{2}=\bar{P}^{-1} s_{2} s_{0} s_{1} s_{2} K_{-2} s_{2} s_{1} s_{0} \bar{P}, \quad S_{0}=\bar{P}^{-1} s_{0} s_{1} s_{2} s_{3} K_{-1} Q s_{3} s_{2} s_{1} P, \\
& S_{3}=\bar{P}^{-1} s_{3} s_{2} s_{0} s_{1} s_{2} K_{-4} s_{2} s_{1} s_{0} s_{2} \bar{P}, \quad S_{2}=\bar{P}^{-1} s_{2} s_{0} s_{1} s_{2} s_{3} K_{-2} s_{3} s_{2} s_{1} s_{0} \bar{P}, \\
& S_{4}=\bar{P}^{-1} s_{4} s_{3} s_{2} s_{0} s_{1} s_{2} K_{4} s_{2} s_{1} s_{0} s_{2} s_{3} \bar{P}, \quad S_{3}=\bar{P}^{-1} s_{3} s_{2} s_{0} s_{1} s_{2} s_{3} K_{-3} s_{3} s_{2} s_{1} s_{0} s_{2} \bar{P}
\end{aligned}
$$




$$
\begin{array}{ll}
a=-4 & a=-3 \\
S_{4}=P^{-1} s_{4} K_{3} P, & S_{3}=P^{-1} s_{3} K_{-4} P \\
S_{2}=P^{-1} s_{2} s_{4} K_{2} s_{4} P, & S_{4}=P^{-1} s_{4} s_{3} K_{4} s_{3} P, \\
S_{1}=P^{-1} s_{1} s_{2} s_{4} K_{1} s_{4} s_{2} P, & S_{2}=P^{-1} s_{2} s_{4} s_{3} K_{2} s_{3} s_{4} P, \\
S_{0}=\bar{P}^{-1} s_{0} s_{1} s_{2} s_{4} K_{-1} Q s_{4} s_{2} s_{1} P, & S_{1}=P^{-1} s_{1} s_{2} s_{4} s_{3} K_{1} s_{3} s_{4} s_{2} P, \\
S_{2}=\bar{P}^{-1} s_{2} s_{0} s_{1} s_{2} s_{4} K_{-2} s_{4} s_{2} s_{1} s_{0} \bar{P}, & S_{0}=\bar{P}^{-1} s_{0} s_{1} s_{2} s_{4} s_{3} K_{-1} Q s_{3} s_{4} s_{2} s_{1} P, \\
S_{4}=\bar{P}^{-1} s_{4} s_{2} s_{0} s_{1} s_{2} s_{4} K_{-3} s_{4} s_{2} s_{1} s_{0} s_{2} \bar{P}, & S_{2}=\bar{P}^{-1} s_{2} s_{0} s_{1} s_{2} s_{4} s_{3} K_{-2} s_{3} s_{4} s_{2} s_{1} s_{0} \bar{P}, \\
a=-2 & \\
S_{2}=P^{-1} s_{2} K_{-3} P, & S_{1}=P^{-1} s_{1} K_{-2} P \\
S_{3}=P^{-1} s_{3} s_{2} K_{-4} s_{2} P, & S_{2}=P^{-1} s_{2} s_{1} K_{-3} s_{1} P, \\
S_{4}=P^{-1} s_{4} s_{3} s_{2} K_{4} s_{2} s_{3} P, & S_{3}=P^{-1} s_{3} s_{2} s_{1} K_{-4} s_{1} s_{2} P, \\
S_{2}=P^{-1} s_{2} s_{4} s_{3} s_{2} K_{3} s_{2} s_{3} s_{4} P, & S_{4}=P^{-1} s_{4} s_{3} s_{2} s_{1} K_{4} s_{1} s_{2} s_{3} P, \\
S_{1}=P^{-1} s_{1} s_{2} s_{4} s_{3} s_{2} K_{1} s_{2} s_{3} s_{4} s_{2} P, & S_{2}=P^{-1} s_{2} s_{4} s_{3} s_{2} s_{1} K_{3} s_{1} s_{2} s_{3} s_{4} P, \\
S_{0}=\bar{P}^{-1} s_{0} s_{1} s_{2} s_{4} s_{3} s_{2} K_{-1} Q s_{2} s_{3} s_{4} s_{2} s_{1} P, & S_{1}=P^{-1} s_{1} s_{2} s_{4} s_{3} s_{2} s_{1} K_{2} s_{1} s_{2} s_{3} s_{4} s_{2} P .
\end{array}
$$

For instance in $a=1$ case, (24) implies that the first $S_{0}$ acts as $W[1]_{l} \rightarrow W[-2]_{l}$ whereas the last $S_{0}$ does as $W[2]_{l} \rightarrow W[-1]_{l}$. Such a distinction has not been exhibited in the left hand sides.

Example 14. The commutative diagram corresponding to $a=3$ case in Example 13.

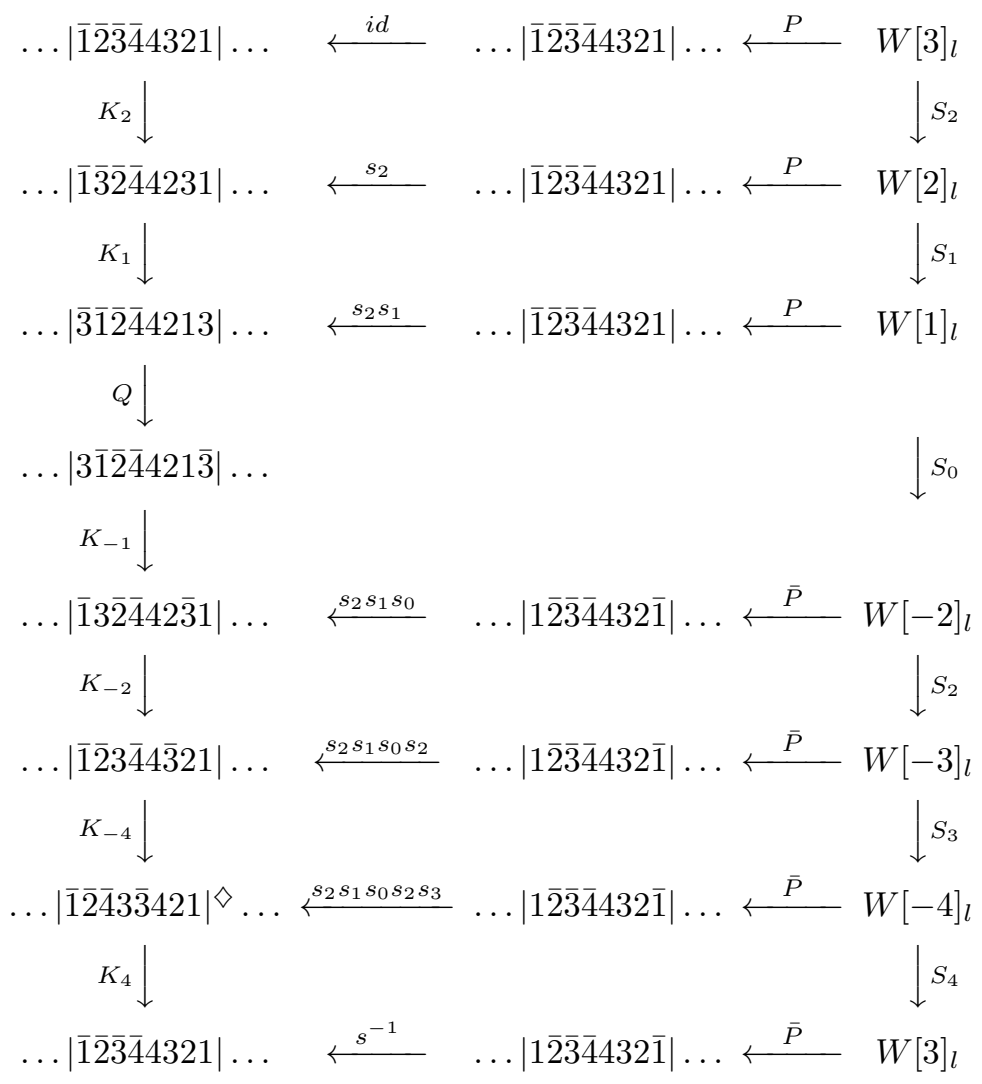


Here, ... $|3 \overline{1} \overline{2} \overline{4} 421 \overline{3}| \ldots$ for example indicates that in all the cells the letters from $J=$ $\{ \pm 1, \ldots, \pm 4\}$ are ordered as

$$
3 \ldots 3-1 \ldots-1-2 \ldots-2-4 \ldots-44 \ldots 42 \ldots 21 \ldots 1-3 \ldots-3 .
$$

In the case with (resp. without) the symbol $\diamond, 3 \overline{3}$ may be written as $\overline{3} 3$ (resp. $\overline{4} 4$ may be written as $4 \overline{4})$ because the cells do not contain these letters simultaneously.

\section{Soliton}

Let us present the configurations that behave as solitons, namely, some special patterns of local states that undergo only translation under the time evolution $\mathcal{T}_{a}=T_{a}$. We concentrate on the basic $D_{n}^{(1)}$-automaton but treat the general boundary condition labeled with an arbitrary $a \in J$. The case $a=1$ was studied in [HKT1]. Solitons in the inhomogeneous $D_{n}^{(1)}$-automaton can then be understood along the argument in section 5 of [HKOTY]. See also section III in [HHIKTT]. Note that the operators $P$ and $Q$ become trivial for the basic automaton, hence they can be dropped in Definition 3.

Fix $a \in J$ which specifies the boundary condition as in (1). Let $i_{1}, \ldots, i_{2 n-2} \in$ $\{0,1, \ldots, n\}$ be any sequence such that $\varepsilon_{i_{t}}\left(r_{i_{t-1}} \cdots r_{i_{1}}(a)\right)>0$ for all $1 \leq t \leq 2 n-2$ and $r_{i_{2 n-2}} \cdots r_{i_{1}}(a)=r(a)$. $(r$ is defined after $(24)$.) Such a sequence exists for any $a$ and corresponds to a path on the crystal graph (Figure 1) going from $a$ to $r(a)$ backward the arrows. Set $a_{t}=r_{i_{t-1}} \cdots r_{i_{1}}(a)$. By the definition $a_{2 n-2}=r(a)$ holds. There is no $a$ and exactly one $-a$ in the array $a_{1}, a_{2}, \ldots, a_{2 n-3}$.

Proposition 15. Consider the following state of the basic $D_{n}^{(1)}$-automaton:

$$
\ldots a \overbrace{\overbrace{a_{1} \ldots a_{1}}^{y_{1}}}^{y_{a_{2} \ldots a_{2}} \ldots \overbrace{a_{2 n-3} \ldots a_{2 n-3}}^{y_{2}}} \overbrace{a_{2 n-2} \ldots a_{2 n-2}}^{y_{2 n-3}} a \ldots \ldots,
$$

where $\ldots$ on the both ends stands for the array of a only, and $y_{1}, \ldots, y_{2 n-2}$ are nonnegative integers satisfying

$$
y_{2 n-2}=\left\{\begin{array}{l}
0 \quad \text { if } a \in\{ \pm 1, \pm n\} \quad(\text { i.e. }, r(a) \neq a), \\
y_{p} \quad \text { otherwise }
\end{array}\right.
$$

where $p$ is determined by $a_{p}=-a$. Under the time evolution $\mathcal{T}_{a}=T_{a}$, the underlined pattern is shifted to the right by $y_{1}+\cdots+y_{2 n-2}$ lattice units.

Proof. The case $a=1$ was proved in [HKT1]. From Definition 1, the time evolutions under the other boundary conditions are connected by a similarity transformation as $\mathcal{T}_{a}=w^{-1} \mathcal{T}_{1} w$, where $w$ is an element of the affine Weyl group. The pattern (30) is obtained by applying $w^{-1}$ to the soliton states [HKT1] for $a=1$.

We call the underlined pattern a soliton with amplitude $y_{1}+\cdots+y_{2 n-2}$. Proposition 15 states that the amplitude coincides with the velocity of solitons. Note that for $a \neq$ $\pm 1, \pm n$, the 'empty box' $a$ is regarded also as a part of a soliton. To include them in the right end of solitons is just a convention. Proposition 15 is equally valid if a

soliton is defined, instead of $(30)$, as ... $\overbrace{a \ldots a}^{\alpha} a_{1}, \ldots a_{2 n-3} \overbrace{a \ldots a}^{\beta} \ldots$ for any $\alpha$ and $\beta$ such that $\alpha+\beta=y_{2 n-2}$. If there are more than one solitons, they move independently as 
described in Proposition 15 as long as they are sufficiently separated from each other. As for their scattering theory, see [HKOTY].

Let us explicitly list the possible choices of the array $a_{1}, a_{2}, \ldots, a_{2 n-3}$ for $a \in\{ \pm 1, \pm n\}$ and $a_{1}, a_{2}, \ldots, a_{2 n-2}$ for $a \in\{ \pm 2, \ldots, \pm(n-1)\}$.

$$
\begin{aligned}
& -2,-3, \ldots-n+1,\left\{\begin{array}{c}
n \\
-n
\end{array}\right\}, n-1, \ldots, 3,2 \quad \text { for } a= \pm 1, \\
& n-1, \ldots, 2,\left\{\begin{array}{c}
1 \\
-1
\end{array}\right\},-2, \ldots,-n+1 \quad \text { for } a= \pm n, \\
& a-1, a-2, \ldots, 2,\left\{\begin{array}{c}
1 \\
-1
\end{array}\right\},-2, \ldots,-n+1,\left\{\begin{array}{c}
n \\
-n
\end{array}\right\}, n-1, \ldots, a+1, a \text { for } 2 \leq a \leq n-1, \\
& a-1, a-2, \ldots,-n+1,\left\{\begin{array}{c}
n \\
-n
\end{array}\right\}, n-1, \ldots, 2,\left\{\begin{array}{c}
1 \\
-1
\end{array}\right\},-2, \ldots, a+1, a \text { for }-n+1 \leq a \leq-2 .
\end{aligned}
$$

In the first line, the symbol $\left\{\begin{array}{c}n \\ -n\end{array}\right\}$ means that both $T_{1}$ and $T_{-1}$ admit two arrays corresponding to the choices of $n$ or $-n$. The second line is similar. In the last two lines, each $T_{a}(a \in\{ \pm 2, \ldots, \pm(n-1)\})$ admits four arrays.

Example 16. Under the time evolution $T_{2}=K_{3} K_{4} K_{-4} K_{-3} K_{-1} K_{1}$ in the basic $D_{4}^{(1)}$ automaton, one has (to save the space we write $\overline{3}$ to mean -3 etc.)

$$
\begin{array}{r}
\ldots 22 \underline{1} \overline{2} \overline{3} 44322222222222222 \ldots \\
\stackrel{K_{1}}{\longmapsto} \ldots 222 \overline{1} \overline{1} \overline{3} 44311122222222222 \ldots \\
\stackrel{K_{-1}}{\longmapsto} \ldots 22222 \overline{3} 4432 \overline{2} 122222222222 \ldots \\
\stackrel{K_{-3}}{\longmapsto} \ldots 22222244 \overline{2} 331 \overline{3} \overline{3} 222222222 \ldots \\
\stackrel{K_{-4}}{\longmapsto} \ldots 222222444331 \overline{3} \overline{3} \overline{4} 22222222 \ldots \\
\stackrel{K_{4}}{\longmapsto} \ldots 222222222331 \overline{3} \overline{3} \overline{2} 44222222 \ldots \\
\stackrel{K_{3}}{\longmapsto} \ldots 22222222222 \underline{1} \overline{2} \overline{2} \overline{3} 44322222 \ldots
\end{array}
$$

A soliton with amplitude 9 (underlined) is shifted to the right by 9 lattice units.

\section{A Crystal structure of $B_{k}$ for $D_{n}^{(1)}$}

Here we quote the $D_{n}^{(1)}$ crystal structure of $B_{k}[\mathrm{KKM}]$ in a form adapted to section 5. In this paper we write the Kashiwara operators $\tilde{e}_{i}$ and $\tilde{f}_{i}(0 \leq i \leq n)$ as $e_{i}$ and $f_{i}$, respectively. Moreover we employ the convention $e_{i}^{q}=f_{i}^{-q}$ for $q<0$. Let $\vec{x}=$ $\left(x_{1}, \ldots, x_{n}, x_{-n}, \ldots, x_{-1}\right)$ be an element of the $D_{n}^{(1)}$-crystal as described in Table 1. For $q \in \mathbb{Z}$, we set $e_{i}^{q}(\vec{x})=\vec{x}^{\prime}$. We assume that $\vec{x}^{\prime} \neq 0$ and let $\Delta x_{j}=x_{j}^{\prime}-x_{j}$ denote the change of the coordinates $(j \in J)$. For each $0 \leq i \leq n$, all the $\Delta x_{j}$ 's are zero except the 
following:

$$
\begin{array}{ll}
i=0 & \left\{\begin{array}{l}
\Delta x_{-1}=-\Delta x_{2}=\left(x_{2}-x_{-2}\right)_{+}-\left(x_{2}-x_{-2}-q\right)_{+}, \\
\Delta x_{1}=-\Delta x_{-2}=\left(x_{-2}-x_{2}\right)_{+}-\left(x_{-2}-x_{2}+q\right)_{+},
\end{array}\right. \\
1 \leq i \leq n-1 & \left\{\begin{array}{l}
\Delta x_{i}=-\Delta x_{i+1}=\left(x_{i+1}-x_{-i-1}\right)_{+}-\left(x_{i+1}-x_{-i-1}-q\right)_{+}, \\
\Delta x_{-i}=-\Delta x_{-i-1}=\left(x_{-i-1}-x_{i+1}\right)_{+}-\left(x_{-i-1}-x_{i+1}+q\right)_{+},
\end{array}\right. \\
i=n & \left\{\begin{array}{l}
\Delta x_{n-1}=-\Delta x_{-n}=x_{-n}-\left(x_{-n}-x_{n}-q\right)_{+}, \\
\Delta x_{-n+1}=-\Delta x_{n}=x_{n}-\left(x_{n}-x_{-n}+q\right)_{+} .
\end{array}\right.
\end{array}
$$

Here we have used the symbol $(z)_{+}=\max (z, 0)$.

If one associates a node to each element $\vec{x} \in B_{k}$ and a figure $\vec{x} \stackrel{i}{\leftarrow} \vec{x}^{\prime}$ to each relation $e_{i}(\vec{x})=\vec{x}^{\prime}$, the result is an oriented graph called a crystal graph, whose arrows carry the colors $0,1, \ldots, n$. For $k=1$, the crystal graph of $B_{1}$ looks as Figure 1 .

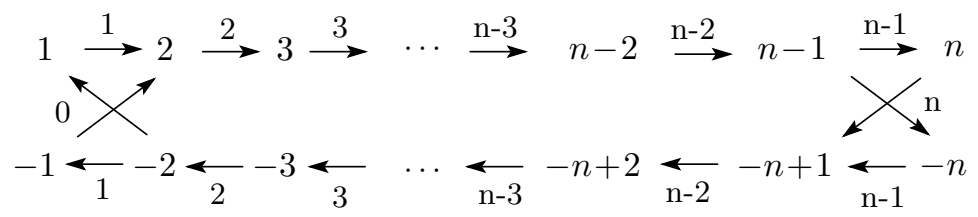

Figure 1: Crystal graph of $B_{1}$

Here the elements of $B_{1}$ are denoted by the letter in $J$. (See the second paragraph of section 3.)

The functions $\varphi_{i}, \varepsilon_{i}: B_{k} \rightarrow \mathbb{Z}_{\geq 0}$ are given by

$$
\begin{aligned}
& \varphi_{0}(\vec{x})=x_{-1}+\left(x_{-2}-x_{2}\right)_{+}, \quad \varepsilon_{0}(\vec{x})=x_{1}+\left(x_{2}-x_{-2}\right)_{+}, \\
& \varphi_{i}(\vec{x})=x_{i}+\left(x_{-i-1}-x_{i+1}\right)_{+}, \quad \varepsilon_{i}(\vec{x})=x_{-i}+\left(x_{i+1}-x_{-i-1}\right)_{+} \quad 1 \leq i \leq n-1, \\
& \varphi_{n}(\vec{x})=x_{n-1}+x_{n}, \quad \varepsilon_{n}(\vec{x})=x_{-n+1}+x_{-n} .
\end{aligned}
$$

Note that $\left(x_{j}-x_{-j}\right)_{+}=x_{j}$ for $j= \pm n$ due to the constraint $x_{n} x_{-n}=0$. For $p \in B_{1}$, one has the simple description

$$
\varepsilon_{i}(p)= \begin{cases}1 & \text { if } p^{\prime} \stackrel{i}{\rightarrow} p \text { for some } p^{\prime} \in B_{1} \\ 0 & \text { otherwise }\end{cases}
$$

The Weyl group operator is defined by $S_{i}=e_{i}^{\varepsilon_{i}-\varphi_{i}}$. Whenever $S_{i}$ acts on the states $\xi \in W[a]_{l}$, it acts as $S_{i}=e_{i}^{\infty}$ since $\varphi_{i}(\xi)=0$ holds due to the boundary condition. In such a situation, $\xi^{\prime}=S_{i}(\xi)$ can be computed recursively as

$$
\begin{aligned}
& \xi=\left(\ldots \vec{\xi}_{g}, \vec{\xi}_{g+1}, \ldots\right), \quad \xi^{\prime}=\left(\ldots \vec{\xi}_{g}^{\prime}, \vec{\xi}_{g+1}^{\prime}, \ldots\right), \\
& \vec{\xi}_{g}^{\prime}=e_{i}^{\left(\varepsilon_{i}\left(\vec{\xi}_{g}\right)-m_{g}\right)_{+}}\left(\vec{\xi}_{g}\right), \quad m_{g+1}=\left(m_{g}-\varepsilon_{i}\left(\vec{\xi}_{g}\right)\right)_{+}+\varphi_{i}\left(\vec{\xi}_{g}\right), \\
& \vec{\xi}_{g}^{\prime}=e_{i}^{\varepsilon_{i}\left(\vec{\xi}_{g}\right)}\left(\vec{\xi}_{g}\right), \quad m_{g}=0 \text { for } g \ll-1 .
\end{aligned}
$$

The $m_{g+1} \in \mathbb{Z}_{\geq 0}$ determined as above is equal to $\varphi_{i}\left(\left(\ldots, \vec{\xi}_{g-1}, \vec{\xi}_{g}\right)\right)$. 


\section{B Proof of Proposition 11}

Here we exhibit the dependence of the operators $K_{j}$ and $Q$ on the boundary condition $a \in J$ as $K_{j}^{(a)}$ and $Q^{(a)}$. By the definition it is easy to see

Lemma 17. For any $0 \leq i \leq n$ and $a, j \in J$, the following is valid:

$$
\begin{aligned}
& s_{i} K_{j}^{(a)}=K_{r_{i}(j)}^{\left(r_{i}(a)\right)} s_{i}, \\
& s_{i} Q^{(a)}=Q^{\left(r_{i}(a)\right)} s_{i} .
\end{aligned}
$$

We denote by $\left.S_{i}\right|_{W[a]_{l}}$ the restriction of $S_{i}$ on $W[a]_{l}$.

\section{Lemma 18.}

$$
\begin{array}{lc}
\left.S_{0}\right|_{W[1]_{l}}=\bar{P}^{-1} s_{0} K_{-2}^{(1)} Q^{(1)} P, & \left.S_{0}\right|_{W[2]_{l}}=\bar{P}^{-1} s_{0} K_{-1}^{(2)} \bar{P}, \\
\left.S_{1}\right|_{W[2]_{l}}=P^{-1} s_{1} K_{1}^{(2)} P, & \left.S_{1}\right|_{W[-1]_{l}}=P^{-1} s_{1} K_{-2}^{(-1)} P, \\
\left.S_{i}\right|_{W[c]_{l}}=P^{-1} s_{i} K_{r_{i}(c)}^{(c)} P=\bar{P}^{-1} s_{i} K_{r_{i}(c)}^{(c)} \bar{P} & (i \neq 0,1),
\end{array}
$$

where in (45), $c$ is any element of $J=B_{1}$ satisfying $\varepsilon_{i}(c)>0$. (For any $i \neq 0,1$, there are two such $c$, which are contained in the crystal graph as $r_{i}(c) \stackrel{i}{\longrightarrow} c$.)

Proof. Let us show $\left.S_{i}\right|_{W[i+1]_{l}}=P^{-1} s_{i} K_{i}^{(i+1)} P$ for $2 \leq i \leq n-1$, which is a part of the assertion (45). The other cases can be verified similarly. Obviously, the map $s_{i} K_{i}^{(i+1)}: \widetilde{W}[i+1]_{l} \rightarrow \widetilde{W}[i]_{l}$ only changes the letters $\pm i, \pm(i+1)$ in each cell. So setting $\vec{\xi}_{g}=\left(x_{1}, \ldots, x_{-1}\right) \in B_{l_{g}}$, we only consider their array within a cell:

$$
\overbrace{-i, \ldots,-i}^{x_{-i}} \overbrace{-i-1, \ldots,-i-1}^{x_{-i-1}} \overbrace{i+1, \ldots, i+1}^{x_{i+1}} \overbrace{i, \ldots, i}^{x_{i}},
$$

dropping the other letters. When $K_{i}^{(i+1)}$ is applied on this array according to the vertex diagram rule (9), it is transformed as

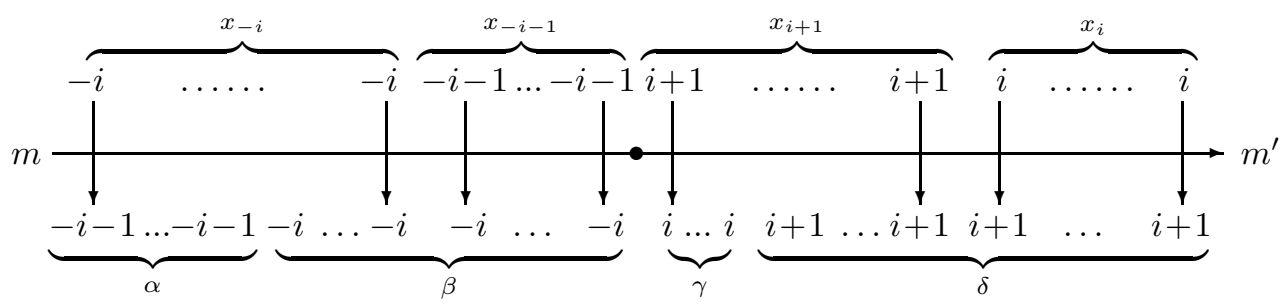

where we have assumed that the number attached to the horizontal line as in (9) is $m$ for the cell in question. Here $\alpha, \beta, \gamma, \delta$ and $m^{\prime}$ are given by

$$
\begin{aligned}
& \alpha=\min \left(x_{-i}, m\right), \quad \beta=x_{-i-1}+\left(x_{-i}-m\right)_{+}, \\
& \gamma=\min \left(x_{i+1}, \tilde{m}\right), \quad \delta=x_{i}+\left(x_{i+1}-\tilde{m}\right)_{+}, \\
& m^{\prime}=x_{i}+\left(\tilde{m}-x_{i+1}\right)_{+}, \quad \tilde{m}=x_{-i-1}+\left(m-x_{-i}\right)_{+},
\end{aligned}
$$

where $\tilde{m}$ is the number attached to the horizontal line at the position marked with the symbol $\bullet$. Thus under $P^{-1} s_{i} K_{i}^{(i+1)} P$, the element $\vec{\xi}_{g}$ is transformed into $\vec{\xi}_{g}^{\prime}=$ $\left(x_{1}^{\prime}, \ldots, x_{-1}^{\prime}\right) \in B_{l_{g}}$ with non-trivial changes given by $x_{-i}^{\prime}=\alpha, x_{-i-1}^{\prime}=\beta, x_{i+1}^{\prime}=\gamma$ and 
$x_{i}^{\prime}=\delta$. We are to show that this coincides with the rule (38)-(40) for $S_{i}$. In view of (32), it is equivalent to checking

$$
\begin{aligned}
& \delta-x_{i}=x_{i+1}-\gamma=\left(x_{i+1}-x_{-i-1}\right)_{+}-\left(x_{i+1}-x_{-i-1}-q\right)_{+}, \\
& \alpha-x_{-i}=x_{-i-1}-\beta=\left(x_{-i-1}-x_{i+1}\right)_{+}-\left(x_{-i-1}-x_{i+1}+q\right)_{+}, \\
& m^{\prime}=\left(m-x_{-i}-\left(x_{i+1}-x_{-i-1}\right)_{+}\right)_{+}+x_{i}+\left(x_{-i-1}-x_{i+1}\right)_{+},
\end{aligned}
$$

where $q=\left(x_{-i}+\left(x_{i+1}-x_{-i-1}\right)_{+}-m\right)_{+}$. These identities can be proved straightforwardly.

Example 19. For $n=4$, the relations (45) read

$$
\begin{aligned}
& \left.S_{2}\right|_{W[3]_{l}}=P^{-1} s_{2} K_{2}^{(3)} P=\bar{P}^{-1} s_{2} K_{2}^{(3)} \bar{P}, \\
& \left.S_{2}\right|_{W[-2]_{l}}=P^{-1} s_{2} K_{-3}^{(-2)} P=\bar{P}^{-1} s_{2} K_{-3}^{(-2)} \bar{P}, \\
& \left.S_{3}\right|_{W[4]_{l}}=P^{-1} s_{3} K_{3}^{(4)} P=\bar{P}^{-1} s_{3} K_{3}^{(4)} \bar{P}, \\
& \left.S_{3}\right|_{W[-3]_{l}}=P^{-1} s_{3} K_{-4}^{(-3)} P=\bar{P}^{-1} s_{3} K_{-4}^{(-3)} \bar{P}, \\
& \left.S_{4}\right|_{W[-4]_{l}}=P^{-1} s_{4} K_{3}^{(-4)} P=\bar{P}^{-1} s_{4} K_{3}^{(-4)} \bar{P}, \\
& \left.S_{4}\right|_{W[-3]_{l}}=P^{-1} s_{4} K_{4}^{(-3)} P=\bar{P}^{-1} s_{4} K_{4}^{(-3)} \bar{P} .
\end{aligned}
$$

Proof of Proposition 11. All the relations (27)-(29) are reduced to Lemma 18 by means of Lemma 17. In fact consider the case $i_{t} \neq 0,1$. Then from Remark 12, the identities to be shown are either (27) or (29). With the aid of Lemma 17, they are reduced to

$$
\left.S_{i_{t}}\right|_{W\left[r_{i_{t-1}} \cdots r_{i_{1}}(a)\right]_{l}}=P^{-1} s_{i_{t}} K_{r_{i_{t-1}} \cdots r_{i_{1}}\left(j_{t}\right)}^{\left(r_{i_{t-1}} \cdots r_{i_{1}}(a)\right)} P \text { or } \bar{P}^{-1} s_{i_{t}} K_{r_{i_{t-1}} \cdots r_{i_{1}}\left(j_{t}\right)}^{\left(r_{i_{t}} \cdots r_{i_{1}}(a)\right)} \bar{P}
$$

where in the left hand side, we have exhibited the fact (24) explicitly. Substitute (23) into this and set $r_{i_{t-1}} \cdots r_{i_{1}}(a)=c$ and $i_{t}=i$. The result coincides with (45). Moreover, the property $(21)$ tells that $\varepsilon_{i}(c)>0$. Thus $(55)$ is reduced to Lemma 18 . The remaining case $i_{t}=0,1$ can be shown similarly by means of (43) and (44). This completes a proof of Proposition 11.

Remark 20. By substituting (45) into $S_{n-1} S_{n}=S_{n} S_{n-1}$ and applying Lemma 17, one can show $K_{n}^{(a)} K_{-n}^{(a)}=K_{-n}^{(a)} K_{n}^{(a)}$ for $a \neq \pm n$. Similarly $S_{0} S_{1}=S_{1} S_{0}$ leads to $Q^{(1)} K_{-1}^{(a)} Q^{(a)} K_{1}^{(a)}=K_{1}^{(a)} Q^{(a)} K_{-1}^{(a)} Q^{(1)}$ for $a \neq \pm 1$.

Finally we include a lemma concerning the operator $K_{-a}$ appearing in the $A_{2 n-3^{-}}^{(2)}$ automaton and the vertex diagram (14).

Lemma 21. If $x_{n}=x_{-n}=0$ for all the local states $\vec{x}=\left(x_{1}, \ldots, x_{-1}\right)$ in the $D_{n}^{(1)}$ automaton, the composition $K_{n} K_{-n}$ appearing in $T_{a}(a \neq \pm n)$ is equal to $K_{-a}$ defined around (14).

Proof. From the remark made after Example 4, the $K_{n} K_{-n}$ always acts on the cells of the form ... $\overbrace{a \ldots a}^{\alpha} \overbrace{-a \ldots-a}^{\beta} \ldots$, where the ... parts on the both ends do not contain the letters $\pm a, \pm n$ and are kept unchanged. Therefore by dropping them, the action of $K_{n} K_{-n}$ on a cell can be computed with the following diagram: 


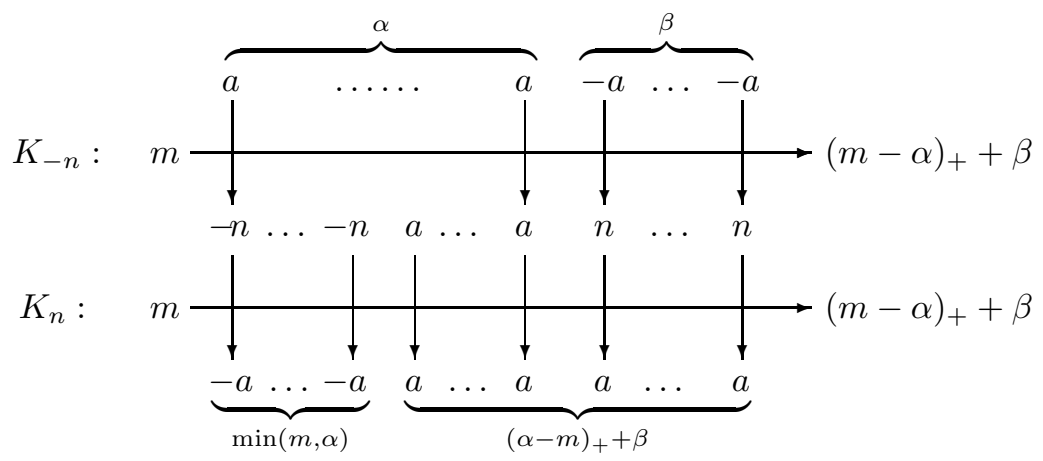

One finds that the letters $\pm n$ are indeed confined within the intermediate state between $K_{n}$ and $K_{-n}$. Moreover, the composition $K_{n} K_{-n}$ induces the same transformation as $K_{-a}$ defined by (14).

Acknowledgements A.K. is partially supported by Grand-in-Aid for Scientific Research JSPS No.15540363.

\section{References}

[F] K. Fukuda, Box-ball systems and Robinson-Schensted-Knuth correspondence, J. Alg. Comb. in press. (math.CO/0105226 v3)

[FOY] K. Fukuda, M. Okado, Y. Yamada, Energy functions in box ball systems, Int. J. Mod. Phys. A 15 (2000) 1379-1392.

[HHIKTT] G. Hatayama, K. Hikami, R. Inoue, A. Kuniba, T. Takagi and T. Tokihiro, The $A_{M}^{(1)}$ Automata related to crystals of symmetric tensors, J. Math. Phys. 42 (2001) 274-308.

[HKOTY] G. Hatayama, A. Kuniba, M. Okado, T. Takagi and Y. Yamada, Scattering rules in soliton cellular automata associated with crystal bases, Contemporary Math. 297 (2002) 151-182.

[HKT1] G. Hatayama, A. Kuniba, and T. Takagi, Soliton cellular automata associated with crystal bases, Nucl. Phys. B577[PM] (2000) 619-645.

[HKT2] G. Hatayama, A. Kuniba, and T. Takagi, Factorization of combinatorial $R$ matrices and associated cellular automata, J. Stat. Phys. 102 (2001) 843-863.

[HKT3] G. Hatayama, A. Kuniba, and T. Takagi, Simple Algorithm for Factorized Dynamics of $\mathfrak{g}_{n}$-Automaton, J. Phys. A: Math. Gen.34 (2001) 10697-10705.

[HIK] K. Hikami, R. Inoue and Y. Komori, Crystallization of the Bogoyavlensky lattice, J. Phys. Soc. Jpn. 68 (1999) 2234-2240.

[KKM] S-J. Kang, M. Kashiwara and K. C. Misra, Crystal bases of Verma modules for quantum affine Lie algebras, Compositio Math. 92 (1994) 299-325.

[K] M. Kashiwara, Crystal bases of modified quantized universal enveloping algebra, Duke Math. 73: 383-413 (1994). 
[KOTY1] A. Kuniba, M. Okado, T. Takagi and Y. Yamada, Geometric crystal and tropical $R$ for $D_{n}^{(1)}$, International Mathematics Research Notices in press. (math.QA/0208239)

[KOTY2] A. Kuniba, M. Okado, T. Takagi and Y. Yamada, Tropical $R$ and tau functions, Commun. Math. Phys. in press. (math.QA/0303088)

[T] D. Takahashi, On some soliton systems defined by using boxes and balls, Proceedings of the International Symposium on Nonlinear Theory and Its Applications (NOLTA '93), (1993) 555-558.

[TM] D. Takahashi and J. Matsukidaira, Box and ball system with a carrier and ultradiscrete modified KdV equation, J. Phys. A 30 (1997) L733 - L739.

[TS] D. Takahashi and J. Satsuma, A soliton cellular automaton, J. Phys. Soc. Jpn. 59 (1990) 3514-3519.

[TTMS] T. Tokihiro, D. Takahashi, J. Matsukidaira and J. Satsuma, From soliton equations to integrable cellular automata through a limiting procedure, Phys. Rev. Lett. 76, (1996) 3247-3250. 